\section{Immortal Person-Time in Studies of Cancer Outcomes}

To THE EDITOR: Immortal person-time arises in an observational study when follow-up time is included in person-time at risk for the study outcome, even though that time precedes the last event required for entry into the study population or satisfaction of an exposure definition. ${ }^{1,2}$ Immune person-time is similar, but it pertains to outcomes other than death. If a study patient were to have incurred the outcome or been censored during immortal or immune person-time, then the patient would not have satisfied the requirements for inclusion in the study or exposure category. A study or exposure category that includes immortal or immune person-time yields a downwardly biased outcome rate and an upwardly biased survival curve. This bias occurs because the accumulated person-time exceeds person-time actually at risk. When comparing rates or survival curves among exposure categories, the net effect of immortal or immune persontime bias may be in any direction.

Examples of research studies affected by immortal or immune person-time appear with some regularity in clinical epidemiology, including in Journal of Clinical Oncology. Consider, for example, a study of genetic determinants of cancer outcomes among patients with cancer. If genotyping is conducted on blood drawn from patients after diagnosis, then person-time between diagnosis and drawing of blood is immortal. Patients who do not survive to give blood cannot be included in the study. The immortal person-time between diagnosis and drawing of blood should not be included in outcome rates or survival analysis. Articles by Lim et $\mathrm{al}^{3}$ and Kiyotani et $\mathrm{al}^{4}{ }^{4}$ who reported studies of modification of tamoxifen effectiveness by CYP2D6 genotype in metastatic and adjuvant settings, respectively, are published examples in which immortal person-time was included in outcome rates.

Nonrandomized studies of adherence to protocols are also often susceptible to immortal person-time bias. For example, in a study of the association between delayed initiation of radiation therapy and breast cancer recurrence, ${ }^{5}$ person-time was accrued in exposure categories defined by time from breast-conserving surgery to initiation of radiation therapy. No woman for whom adjuvant radiation therapy was initiated would have had a recurrence between her diagnosis and start of radiation therapy. Time between breast-conserving surgery and initiation of radiation therapy therefore resulted in immortal or immune person-time and should have been excluded from the followup period.

To diagnose the presence of immortal or immune person-time, one can ask: "From the perspective of the study, is the patient at risk for the outcome of interest (or any censoring events) at this exposure level in all of the contributed person-time?" If the answer is yes, then the person-time is mortal and nonimmune and therefore should be counted at that exposure level. But if the answer is no, then at least a portion of the person-time is either immortal or immune, and steps should be taken to account for the immortal or immune person-time.
We illustrate the potential for highly misleading results attributable to inclusion of immortal person-time using a hypothetic study of 5 -year survival after treatment initiation among patients diagnosed at a single clinical center. Suppose investigators prospectively enrolled 350 patients per year for 2 years and observed these patients for 5 years. To bolster sample size, investigators augmented these prospective data with prevalent cases identified retrospectively in the center's registry of current patients. Imagine that they included 350 patients per year for the 5 years before study onset. For each patient with cancer, the investigators knew the number of years from treatment initiation to study enrollment (if any), the number of years from treatment initiation to death, and whether the patient was identified prospectively or retrospectively.

Suppose the 1,750 prospectively enrolled patients with cancer contributed 3,473 person-years at risk for death, during which 111 deaths occurred, yielding a rate of 3.2 deaths per 100 person-years. In addition, 1,750 retrospectively enrolled patients with cancer contributed 8,506 person-years between treatment initiation and end of study; 4,169 person-years (49\%) were accrued after study enrollment, during which 136 deaths occurred. Note that retrospectively enrolled patients had to be current patients at the time the study began, so they had to have survived from diagnosis to study inception. Counting all person-time, both mortal and immortal, among retrospectively enrolled patients would yield an overall rate of 1.6 deaths per 100 person-years, which is approximately half the prospectively measured rate. However, counting only mortal persontime among retrospectively enrolled patients would yield the correct rate of 3.3 deaths per 100 person-years.

In a given study, immortal person-time usually accrues between the first event required for study entry (eg, cancer diagnosis or inception of treatment) and the last event required for study entry (eg, provision of written informed consent or collection of a biologic specimen). Immortal person-time may also arise when a list of potential study patients is used to define a cohort, but the list is modified between the time of its creation and the time it reaches the hands of investigators. For example, if study patients were removed from a registry because of death or emigration, then the registry list would only include survivors at the time it reached investigators. For the survivors on the list, the time between their entry into the registry and delivery of the list to investigators would be immortal person-time.

Accrual of person-time from the date of the last study or exposure-defining criterion thus provides a simple solution for avoiding immortal and immune person-time bias when estimating outcome rates, rate differences, and rate ratios. Allowing patients into risk sets only when person-time is not immortal or immune using methods for late entry ${ }^{6,7}$ or time-varying exposure ${ }^{8}$ provides the analogous solution when estimating survival curves or hazard ratios. This solution of restricting or reallocating counted person-time is not foolproof. Analogous to informative censoring, bias will persist if the timing of late entry is informative with respect to the outcome of interest, conditional on measured variables. ${ }^{9}$ In addition, this solution is not optimal if a study samples exposed and unexposed patients 
differentially from the underlying population. ${ }^{10}$ Immortal and immune person-time can thus be a subtle bias to diagnose, but it can also be as treacherous as it is subtle, and therefore it must be treated rather than overlooked.

\section{Timothy L. Lash}

Department of Epidemiology, Boston University School of Public Health, Boston, MA

\section{Stephen R. Cole}

Department of Epidemiology, Gillings School of Global Public Health, University of North Carolina, Chapel Hill, NC

\section{AUTHORS' DISCLOSURES OF POTENTIAL CONFLICTS OF INTEREST}

The author(s) indicated no potential conflicts of interest.

\section{REFERENCES}

1. Rothman KJ, Greenland S: Cohort studies, in Rothman KJ, Greenland S, Lash TL (eds): Modern Epidemiology (ed 3). Philadelphia, PA, Lippincott Williams \& Wilkins, 2008, pp 106-107

2. Suissa S: Immortal time bias in pharmacoepidemiology. Am J Epidemiol $167: 492-499,2008$
3. Lim HS, Ju Lee $H$, Seok Lee $K$, et al: Clinical implications of CYP2D6 genotypes predictive of tamoxifen pharmacokinetics in metastatic breast cancer $\mathrm{J}$ Clin Oncol 25:3837-3845, 2007

4. Kiyotani K, Mushiroda T, Sasa M, et al: Impact of CYP2D6*10 on recurrence-free survival in breast cancer patients receiving adjuvant tamoxifen therapy. Cancer Sci 99:995-999, 2008

5. Olivotto IA, Lesperance ML, Truong PT, et al: Intervals longer than 20 weeks from breast-conserving surgery to radiation therapy are associated with inferior outcome for women with early-stage breast cancer who are not receiving chemotherapy. J Clin Oncol 27:16-23, 2009

6. Brenner $\mathrm{H}$ : Long-term survival rates of cancer patients achieved by the end of the 20th century: A period analysis. Lancet 360:1131-1135, 2002

7. Lamarca R, Alonso J, Gómez G, et al: Left-truncated data with age as time scale: An alternative for survival analysis in the elderly population. J Gerontol A Biol Sci Med Sci 53:M337-M343, 1998

8. Anderson JR, Cain KC, Gelber RD: Analysis of survival by tumor response and other comparisons of time-to-event by outcome variables. J Clin Oncol 26:3913-3915, 2008

9. Wang MC, Brookmeyer R, Jewell NP: Statistical models for prevalent cohort data. Biometrics 49:1-11, 1993

10. Rothman KJ, Suissa S: Exclusion of immortal person-time. Pharmacoepidemiol Drug Saf 17:1036, 2008

DOI: 10.1200/JCO.2009.24.1877; published online ahead of print at www.jco.org on July 13, 2009 